\title{
Síndrome de Poland y Alteración de la Migración Neuronal: Reporte de un Caso y Revisión de la Literatura
}

\author{
M. JOSEFINA JIMÉNEZ C. ${ }^{1}$, M. JOSÉ LUQUE H. ${ }^{2}$, ELISA JIMÉNEZ A. ${ }^{3}$, TERESA ARAVENA C. ${ }^{4}$ \\ 1. Interna, Escuela de Medicina, Pontificia Universidad Católica de Chile. \\ 2. Interno, Escuela de Medicina, Pontificia Universidad Católica de Chile. \\ 3. Médico Neonatólogo, Servicio de Recién Nacidos, Hospital Dr. Sótero del Río. \\ 4. Médico Genetista, Genética, Hospital Dr. Sótero del Río.
}

\begin{abstract}
Poland Syndrome and Neuronal Migration: Case Report and Review of the Literature

Poland Syndrome is a rare congenital alteration. It is characterized by hypoplasia or absence of the pectoral muscle, hypoplasia or aplasia of the mammary gland, nipple, ribs or cartilages, and hand malformations. A case is reported of a girl showing these malformations, and neuronal migration abnormalities. Emphasis is made upon the need for a multidisciplinary team for management and rehabilitation. A literature review is presented, including pathogenesis, clinical manifestations and treatment.

(Key words: Poland's Syndrome, focomelia, hypoplasia of the pectoral one).

Rev Chil Pediatr 2009; 80 (5): 451-458
\end{abstract}

\section{RESUMEN}

El Síndrome de Poland es una alteración congénita de baja frecuencia y de carácter esporádico. Se caracteriza por ausencia o hipoplasia del músculo pectoral; hipoplasia o aplasia de glándula mamaria, pezón, costillas o cartílagos, y malformaciones de la mano. Se reporta el caso de una recién nacida con Secuencia de Poland con trastorno de migración neuronal. Se hace énfasis en la necesidad de un equipo multidisciplinario en su manejo y rehabilitación. Se presenta una revisión de la literatura con especial énfasis en la patogenia, manifestaciones clínicas y tratamiento.

(Palabras clave: Síndrome de Poland, focomelia, hipoplasia del pectoral).

Rev Chil Pediatr 2009; 80 (5): 451-458

Trabajo recibido el 09 de diciembre de 2008, devuelto para corregir el 12 de enero de 2009, segunda versión el 18 de mayo de 2009, aceptado para publicación el 15 de julio de 2009. 


\section{Introducción}

El síndrome de Poland, conocido también como Secuencia de Poland o anomalía de Poland, fue descrito por primera vez por Alfred Poland en $1841^{1}$. Corresponde a una alteración musculoesquelética congénita caracterizada por la ausencia total o parcial del músculo pectoral mayor asociada a anormalidades de la extremidad superior ipsilateral.

$\mathrm{Su}$ incidencia varía entre 1 en 7000 a 1 en 100000 recién nacidos vivos y en el $75 \%$ de los casos la anomalía se sitúa en el hemicuerpo derecho ${ }^{2}$.

No existe un patrón de herencia determinado, ni factores de riesgo definidos y es excepcional la recurrencia familiar ${ }^{2}$. La patogenia no es bien conocida, aunque se atribuye a una interrupción del flujo sanguíneo de la arteria subclavia durante la sexta semana de gestación ${ }^{3}$.

El diagnóstico se realiza en base a la presencia de aplasia o hipoplasia del músculo pectoral mayor y al menos una anormalidad asociada. Entre éstas, las más frecuentes son aplasia o hipoplasia del pezón, malformaciones de la mano como sindactilia, braquisindactilia, asimetría de extremidad superior, hipoplasia del radio/cúbito, aplasia/hipoplasia costal, ausencia de vello axilar, anormalidades dermatológicas, dextrocardia, anormalidades hepáticas o de vía biliar $^{2,4}$. La presencia de estas alteraciones es variable y excepcionalmente se presenten todas en un mismo individuo.

El objetivo de este trabajo es presentar la historia clínica de una paciente con Síndrome de Poland y alteración de la migración neuronal, y realizar revisión de la literatura, con especial énfasis en la etiopatogenia y manifestaciones clínicas de esta enfermedad.

\section{Caso Clínico}

Recién nacido de término, sexo femenino, segundo hijo de padres jóvenes y sanos, sin antecedentes familiares de importancia ni contacto con teratógenos durante el embarazo. Nació vía parto vaginal con un peso $3340 \mathrm{~g}$, talla de $51 \mathrm{~cm}$, perímetro craneano de $35 \mathrm{~cm}$,
Test de Apgar 8 al minuto y de 9 a los 5 minutos.

Al examen físico destacó un tórax asimétrico por presencia de hipoplasia del pectoral mayor izquierdo, focomelia de brazo izquierdo con sólo 2 dígitos (figuras 1 y 2) micrognatia, clinodactilia. A los minutos de vida tuvo una hipoglicemia severa con convulsiones secundarias que se controlaron con suero glucosado endovenoso y fenobarbital, sin repetir nuevos episodios. Desde que nació fue evidente un examen neurológico alterado con hipotonía central y reflejos osteotendíneos hiperactivos, succión lenta y descoordinada. Tuvo un ritmo bajo de crecimiento del perímetro craneano $(1,5 \mathrm{~cm}$ en 45 días desde el nacimiento) y del incremento ponderal. Además, presentó un síndrome febril hasta $38^{\circ} \mathrm{C}$ en forma cíclica, sin compromiso del estado general con hipernatremia secundaria debido a aumento de pérdidas insensibles. El estudio de infección fue normal y su fiebre interpretada como secundario a probable déficit de ADH en un paciente que tiene alteraciones de imagen del Sistema Nervioso Central. Recibió Diazepam como relajante muscular con buena respuesta.

Dentro del estudio destaca un cariotipo 46,XX normal, radiografía de tórax normal, radiografía de columna con escoliosis moderada, ecografía renal y abdominal normal, ecocardiografía con comunicación interauricular tipo osteum secundum de 4-5 mm y ductus arterioso persistente pequeño restrictivo (figura 3 ). El estudio de osmolaridad plasmática de 315,53 mOsm y natremia $150 \mathrm{mEq} / \mathrm{L}$ (manteniéndose siempre en rango alto) fueron patológicos. La ecografía cerebral con ventriculomegalia leve con septum pellucidum amplio, cuerpo calloso presente y vermix cerebeloso amplio; el scanner cerebral mostró megacisterna magna amplia, leve dilatación de sistema ventricular, escasos surcos sugerente de trastorno de migración neuronal. La resonancia nuclear magnética (RNM) de cerebro mostró un leve aumento de la amplitud del sistema ventricular supratentorial, lesiones de aspecto isquémico parietooccipitales bilaterales y frontales parasagitales con signos de necrosis laminar cortical, núcleos grises centrales normales. Imágenes sugerentes de probable trastorno de migración neuronal. Electroencefalograma y potencial evocados auditivo auto- 


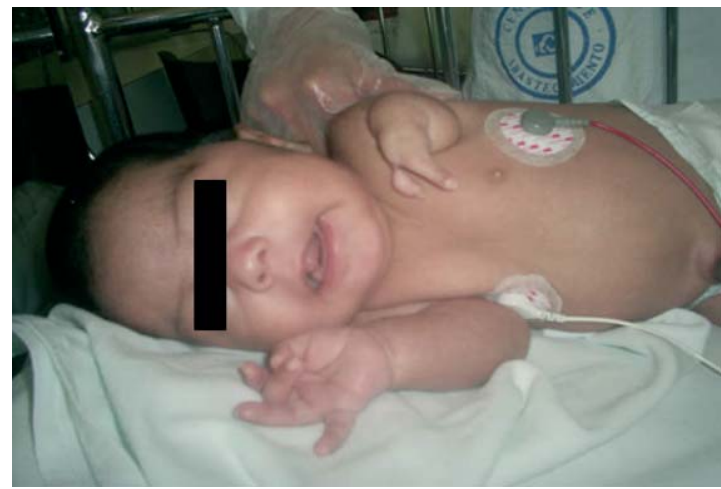

Figura 1. Síndrome de Poland con tórax asimétrico por hipoplasia del pectoral mayor izquierdo.

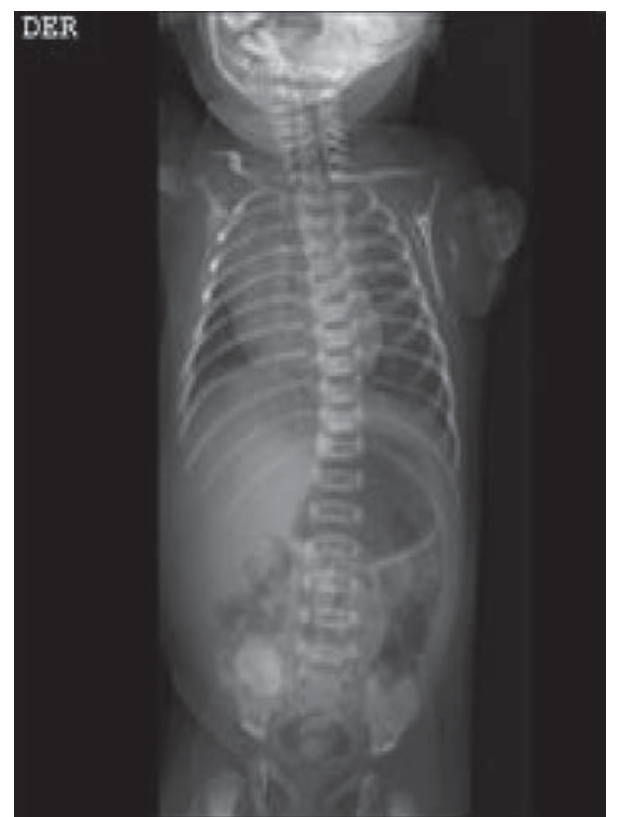

Figura 3. Columna con escoliosis moderada y visualización de focomelia.

matizado (AABR) normales con un potencial evocado visual no concluyente.

Alta a los 43 días de vida con un peso de $3910 \mathrm{~g}$, talla $51,5 \mathrm{~cm}$, y perímetro craneano $37,5 \mathrm{~cm}$, alimentándose por chupete y pecho con buena respuesta. Además se indicó tratamiento con diazepam y fenobarbital. En seguimiento, neurología confirma el diagnóstico de

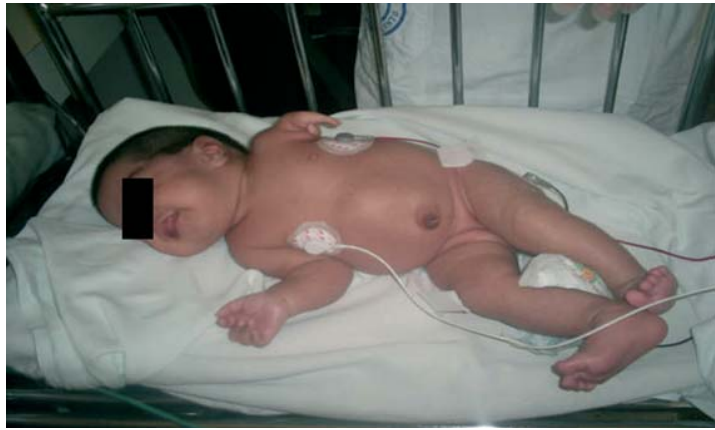

Figura 2. Síndrome de Poland con focomelia de brazo izquierdo.

trastorno de migración neuronal observando un notable incremento en el tono muscular con microcefalia, retraso en el desarrollo psicomotor severo y sin nuevos episodios de convulsiones ni fiebre. Los potenciales evocados visuales confirman visión sólo de luz/sombra y cardiología el cierre del ductus arterioso persistente. No se comprobó déficit de ADH y no repitió episodio de hipernatremia. Ha tenido una hospitalización por neumonía en pediatría. Actualmente, al año de vida, sigue en Nineas (Organización para cuidado de niños especiales) que juega un rol fundamental en la evaluación y rehabilitación de nuestra paciente dado las secuelas neurosensoriales que presenta.

Durante los años 2007 y 2008 hubo 11 casos de Síndrome de Poland en el Hospital Dr. Sótero del Río lo que representó una incidencia de 0,78 por 1000 recién nacidos vivos. Todos los casos fueron esporádicos dentro de las familias La proporción por sexo entre hombres y mujeres fue de 7:4 respectivamente El 100\% presentó agenesia del pectoral mayor. La lateralidad derecha e izquierda fue semejante afectando a 6 recién nacidos a derecha y a 5 en la zona izquierda. Hubo manifestaciones menos frecuentes en orden de presentación como alteración de una extremidad y agenesia de areola (4 y 3 respectivamente). Malformaciones costales, vertebrales y Síndrome de Moebius fueron mucho menos frecuente. A diferencia del caso presentado, ningún paciente presentó alteración de migración neuronal. 


\section{Discusión}

El síndrome de Poland es una alteración musculoesquelética congénita muy poco frecuente, que se caracteriza esencialmente por hipoplasia unilateral de la pared torácica y anormalidades de la extremidad superior ipsilateral. Según lo descrito en la literatura, es considerada una patología de naturaleza esporádica. Es dos a tres veces más frecuente en el sexo masculino, y afecta con mayor frecuencia el lado derecho del cuerpo $(60-75 \%)^{2,5-7}$.

En el año 1841 Alfred Poland, describe la ausencia unilateral de pectoral mayor, serrato anterior y oblicuo externo abdominal, asociado a sindactilia ipsilateral ${ }^{8,1}$.

Su presentación clínica es muy variable, con distintos grados de extensión y severidad del defecto ${ }^{4}$. Es extremadamente raro que todas las anomalías se encuentren presentes en un mismo paciente ${ }^{2,4}$. El rasgo clínico común en todos los pacientes es la ausencia de la porción esternocostal del músculo pectoral mayor, asociado la mayoría de las veces a la ausencia del pectoral menor también ${ }^{2}$. En algunos casos los músculos dorsal ancho, serrato anterior y oblicuo externo abdominal también pueden estar comprometidos.

Además, pueden estar presentes otras alteraciones de la pared torácica, como la hipoplasia o ausencia de la glándula mamaria, aréola y pezón, que afecta a más de un tercio de las pacientes $^{2,9}$. Se han descrito también pezones supernumerarios e hipoplasia o aplasia de las costillas o cartílagos. La aplasia de las porciones anteriores de una a tres costillas, con una depresión severa del tórax, ocurre en un $11-25 \%$ de los pacientes con síndrome de Poland ${ }^{2,9-11}$. En algunos casos los extremos costales de las costillas aplásticas pueden estar fusionadas, produciendo una rotación del esternón hacia el lado comprometido, generando un pectum carinatum asimétrico contralateral ${ }^{2,10}$. Generalmente, la progresión de la deformidad torácica ocurre durante el período de crecimiento. Otros hallazgos al examen físico pueden ser: alopecia de la región mamaria y axilar, e hipoplasia de la piel y tejido subcutáneo a nivel de la pared anterior del tórax ${ }^{2,12}$.

En un $8 \%$ de los pacientes puede haber herniación del pulmón a través del defecto torácico $^{2,12}$. Esto conlleva a un movimiento paradójico de la pared torácica que puede ser muy significativo en casos de estrés o infecciones pulmonares, asociado además a una disminución de la capacidad vital, la cual puede ser tan baja como un $48 \%$ del valor normal ${ }^{2,13}$.

Existen escasos reportes de pacientes con síndrome de Poland y dextrocardia asociada, con una incidencia que alcanza el $5 \%$ de los pacientes con Poland ${ }^{2,3,14}$. A diferencia de los casos de dextrocardia aislada, en los cuales suelen haber otras alteraciones cardiovasculares asociadas, en los pacientes con síndrome de Poland y dextrocardia, ésta se caracteriza porque no se acompaña de situs inversus ni tampoco de otras anomalías cardíacas ${ }^{14}$. En todos estos pacientes con dextrocardia, el defecto de pared torácica compromete costillas del lado izquierdo $^{2,15}$. Se postula que la dextrocardia podría ser consecuencia de una alteración en el desarrollo vascular o secundario a la ocupación de espacio en el hemitorax izquierdo ${ }^{2}$. Es importante destacar que el pronóstico de estos pacientes en general es bueno, ya que no tienen otras malformaciones cardíacas asociadas.

Las malformaciones de la mano afectan a un $13,5 \%$ a $56 \%$ de los pacientes con síndrome de Poland, y estas incluyen: hipoplasia o aplasia de las falanges media y distal, sindactilia simple o compleja (fusión de uno o más dígitos, sin y con compromiso óseo respectivamente), y en casos más severos incluso puede haber ausencia de la mano. También puede haber braquisindactilia e hipoplasia o ausencia de radio, cúbito, húmero y escápula. Es extremadamente raro que la mano afectada sea contralateral al defecto torácico y que haya compromiso de la extremidad inferior ${ }^{2,16-18}$. Ya que existe un amplio rango de anormalidades de la mano que pueden estar presentes, es que algunos autores han propuesto sistemas de clasificación de las malformaciones de mano en pacientes con síndrome de Poland ${ }^{19}$.

El síndrome de Poland también puede coexistir con otras patologías más raras. Una de las asociaciones más conocidas es con el síndrome de Moëbius (15\% de los pacientes), caracterizado por el compromiso de los pares craneales VI y VII, resultando en una parálisis 
facial congénita y estrabismo convergente ${ }^{2,20-23}$. Existen casos reportados de otras asociaciones con alteraciones neurológicas como miastenia gravis $^{24}$, mielomeningocele ${ }^{25}$, parestesia de extremidades $^{26}$ y microcefalia ${ }^{27}$. No hay reportes con asociación de síndrome hipotónico, trastornos de la deglución, trastornos endocrinos, síndromes febriles específicos que pudieran explicar la evolución de nuestra paciente. Los trastornos de la migración neuronal cerebral no están descritos en la literatura lo que hace especialmente diferente este reporte clínico. Se desconoce si existe algún factor común en la etiología de estas patologías. Sí se ha demostrado asociación con alteraciones renales, como agenesia renal unilateral, doble sistema pielocaliciliario y reflujo vesicoureteral, lo cual se denomina defecto acro-pectoral-renal y puede finalmente desencadenar hipertensión de causa renal $1^{2,28,29}$.

El síndrome de Klippel-Feil, que se caracteriza por una fusión de las vértebras cervicales y alteraciones cerebrales, también se ha visto asociado a síndrome de Poland en algunos pacientes $^{2,3}$.

La deformidad de Sprengel o escápula ala$\mathrm{da}$, se puede ver en algunos pacientes con síndrome de Poland que no tienen la porción superior del músculo serrato anterior, lo cual produce una elevación y cabalgamiento de la escápula ${ }^{2,3}$. Finalmente, se ha podido demostrar una relación entre el síndrome de Poland y distintos tumores, entre los que se han descrito destacan: leucemia, linfoma no Hodgkin, cáncer cervical, leiosarcoma, cáncer pulmonar y carcinoma ductal invasor en la mama hipoplásica $^{2,30-32}$.

Excepcionalmente se han descrito asociaciones con otros síndromes como Goldenhar, Adams-Oliver y Parry-Romberg. Se postula que el factor común entre el síndrome de Poland y todas estas patologías, es la presencia de anomalías vasculares que alteran el flujo sanguíneo durante el desarrollo fetal a nivel del defecto respectivo, acuñándose el término SASDS (subclavian artery supply disruption sequence) ${ }^{22}$. Así por ejemplo, en el caso del síndrome de Klippel-Feil existiría un retraso en el desarrollo de las arterias vertebrales, y en el síndrome de Moëbius habría una regresión prematura de las arterias trigeminales primitivas.

Debido a que existe una serie de malformaciones que pueden coexistir junto al síndrome de Poland, se sugiere que todo paciente con el diagnóstico de síndrome de Poland se realice los siguientes exámenes: radiografía de tórax y extremidades superiores, ecocardiograma, ecografía abdominal y renal, hemograma y análisis de orina periódicos ${ }^{33}$.

El reporte de un caso síndrome de Poland en una gemela idéntica, respalda la naturaleza esporádica de la enfermedad ${ }^{2,34}$. Existen escasos reportes de transmisión familiar del defecto, de padres a hijo o de hermanos afectados con padres normales ${ }^{2,35-40}$. Al parecer esta transmisión vertical se debe a una mutación tardía de un gen autosómico dominante, la cual aparentemente se asocia a una mayor edad parental $2,31,36,38-40$. El riesgo de recurrencia en la misma familia es muy bajo, menor al $1 \% 0^{2,41}$.

La etiopatogenia de este síndrome aún no esta clara. El origen vascular del síndrome de Poland constituye la teoría más aceptada hasta el momento. Ésta postula que entre la sexta y séptima semana de gestación ocurre un defecto del mesodermo que resulta en hipoplasia o estenosis de la arteria subclavia en su porción proximal o sus ramas, derivando en un déficit temprano de flujo sanguíneo a nivel de la región pectoral y extremidad de ese lado, que produce disminución parcial o total del tejido en esta región, y así mismo, la hipoplasia de la arteria braquial seria responsable de las alteraciones en la extremidad superior y mano. La localización y grado de la obstrucción de la arteria subclavia, puede determinar la extensión y severidad de las manifestaciones clínicas ${ }^{2,3,42,43}$.

Otras hipótesis que intentan explicar la etiopatogenia del síndrome de Poland incluyen: la interrupción de la placa lateral del mesodermo (a partir de la cual se desarrolla el músculo pectoral $)^{2,44}$; injurias intrauterinas que resultan en trombosis o embolias placentarias que afectan el flujo sanguíneo y alteran la embriogénesis; vasoespasmo inducido por drogas como la cocaína (en esta paciente no existía el antecedente de consumo de drogas) $)^{45}$; herencia autosómica dominante; defectos genéticos simples; trauma; infecciones virales; y efectos terato- 
génicos de xenobióticos ambientales ${ }^{2,7,46}$. Conjuntamente, se ha propuesto que el fumar durante el embarazo puede duplicar el riesgo de síndrome de Poland ${ }^{2,47}$.

La mayoría de los pacientes con síndrome de Poland no requieren de un tratamiento quirúrgico para reparar la deformación torácica. De este modo, la indicación quirúrgica se reserva para los siguientes casos: 1) depresión torácica unilateral con riesgo de progresar; 2) falta de protección del pulmón o corazón; 3) movimiento paradójico de la pared torácica; 4) hipoplasia o aplasia mamaria en mujeres; 5) defecto cosmético secundario a la ausencia del pectoral mayor en hombres ${ }^{2}$.

La reconstrucción o estabilización de las costillas aplásicas se puede conseguir mediante injertos óseos y/o mallas protésicas. El defecto muscular puede ser corregido mediante la trasposición del músculo dorsal ancho y su piel hacia la región anterior de pared torácica. El uso de prótesis mamarias es muy útil para la corrección de la hipoplasia mamaria en el caso de las mujeres, y también para hombres en los cuales existe una asimetría pectoral notoria, ayudando así a conseguir un mejor resultado estético ${ }^{2,10}$.

La reparación quirúrgica de las malformaciones de la mano debe realizarse tempranamente, antes que el paciente desarrolle patrones funcionales anormales o progrese la deformidad. Dependiendo del tipo y grado del defecto va a ser el tipo de cirugía efectuada.

Este caso clínico tiene una presentación diferente a los pacientes que actualmente están en seguimiento en nuestro Servicio y a lo usualmente descrito en la literatura. Como Síndrome es de baja frecuencia pero el espectro de presentación es muy amplio, pudiendo presentarse como una agenesia del pectoral mayor hasta un compromiso neurosensorial severo que lleve a la necesidad de un equipo multidisciplinario para su manejo.

\section{Agradecimientos}

Agradecimientos especiales por su colaboración en la revisión de este caso clínico a Dra. Patricia Mena Nanning, Profesor adjunto de la
Universidad Católica y Jefe de Servicio Recién Nacidos de Hospital Dr. Sótero del Río

\section{Referencias}

1.- Poland A: Deficiency of the pectoral muscles. Guy Hosp Rep 1841; 6: 191-3.

2.- Fokin AA, Robicsek F: Poland.s syndrome revisited. Ann Thorac Surg 2002; 74: 2218-25.

3.- Bavinck $J N$, Weaver DD: Subclavian artery supply disruption sequence: hypothesis of a vascular etiology for Poland, Klippel-Feil, and Möbius anomalies. Am J Med Genet 1986; 23 (4): 903-18.

4.- Urschel HC Jr: Poland's syndrome. Chest Surg Clin North Am 2000; 10: 393-403.

5.- Pérez Aznar JM, Urbano J, García Laborda E, Quevedo Moreno P, Ferrer Vergara L: Breast and pectoralis muscle hypoplasia. A mild degree of Poland's syndrome. Acta Radiol 1996; 37 (5): 75962.

6.- McGillivray BC, Lowry RB: Poland syndrome in British Columbia: incidence and reproductive experience of affected persons. Am J Med Genet 1977; 1 (1): 65 74.

7.- Freire-Maia N, Chautard EA, Opitz JM, Freire-Maia A, Quelce-Salgado A: The Poland syndrome-clinical and genealogical data, dermatoglyphic analysis, and incidence. Hum Hered 1973; 23 (2): 97-104.

8.- Perlyn C, Edmiston J, Tunnessen WW Jr: Picture of the month. Unilateral amastia (Poland syndrome). Arch Pediatr Adolesc Med 1999; 153 (12): 1305-6.

9.- Walker JC Jr, Meijer R, Aranda D: Syndactylism with deformity of the pectoralis muscle. Poland's syndrome. J Pediatr Surg 1969; 4 (5): 569-72.

10.- Shamberger RC, Welch KJ, Upton J 3rd: Surgical treatment of thoracic deformity in Poland's syndrome. J Pediatr Surg 1989; 24 (8): 760-5; discussion 766.

11.- Mace JW, Kaplan JM, Schanberger JE, Gotlin RW: Poland's syndrome. Report of seven cases and review of the literature. Clin Pediatr (Phila) 1972; 11 (2): 98-102.

12.- Ravitch MM: Poland's syndrome. In: Ravitch MM, ed. Congenital deformities of the chest wall and their operative correction. Philadelphia, London, Toronto: WB Saunders 1977: 233-71.

13.- Martin LW, Helmsworth JA: The management of congenital deformities of the sternum. JAMA 1962; 179: 82-4.

14.- Pérez E, Ochoa C, Marugán V, Casanueva T, Carrascal 
A: Secuencia de Poland y dextrocardia. An Pediatr Barc 2004; 61 (4): 344-52.

15.- Fraser FC, Teebi AS, Walsh S, Pinsky L: Poland sequence with dextrocardia: which comes first? Am J Med Genet 1997; 73 (2): 194-6.

16.- Ireland DC, Takayama N, Flatt AE: Poland's syndrome. J Bone Joint Surg Am 1976; 58 (1): 52-8.

17.- Powell CV, Coombs RC, David TJ: Poland anomaly with contralateral ulnar ray defect. J Med Genet 1993; 30 (5): 423-4.

18.- Silengo M, Lerone $M$, Seri M, Boffi P: Lower extremity counterpart of the Poland syndrome. Clin Genet 1999; 55 (1): 41-3.

19.- Al-Qattan MM: Classification of hand anomalies in Poland's syndrome. Br J Plast Surg 2001; 54 (2): 1326.

20.- Koroluk LD, Lanigan DT: Moebius and Poland syndromes: a report of a case. J Can Dent Assoc 1989; 55 (8): 647-8.

21.- Sugarman GI, Stark HH: Möbius syndrome with Poland's anomaly. J Med Genet 1973; 10 (2): 192-6.

22.- Aravena T, Cares C: Asociación sindromatica: Poland, Goldenhar, Moëbius, Klippel Feil. Presentación de un caso clínico. Datos no publicados. Presentado en el Congreso de Pediatría 2006.

23.- Kuklik M: Poland-Möbius syndrome and disruption spectrum affecting the face and extremities: a review paper and presentation of five cases. Acta Chir Plast 2000; 42 (3): 95-103.

24.- Padua-Gabriel A, Navarro-Reynoso F, Cicero-Sabido $R$ : Poland syndrome and myasthenia gravis. Rev Invest Clin 1989; 41 (1): 63-5.

25.- Rattan KN, Budhiraja S, Pandit SK: Poland's syndrome with meningomyelocele. Indian J Chest Dis Allied Sci 1996; 38 (4): 259-61.

26.- Selva A, Len O, Jiménez-Moreno X, Mollet J, Solans $R$, Vilardell $M$ : A 66-year-old man with impaired gait and paresthesias of the lower limbs. Rev Clin Esp 1998; 198 (4): 255-7.

27.- Fryns JP, De Smet L: Follow-up report on the 'Poland anomaly/primary microcephaly association. Clin Dysmorphol 1997; 6 (1): 95-6.

28.- Hegde HR, Leung AK: Aplasia of pectoralis major muscle and renal anomalies. Am J Med Genet 1989; 32 (1): 109-11.

29.- Pranava VM, Rao PS, Neelachalam A, Sailendra VH: Poland's syndrome-complicated with renal hypertension. J Assoc Physicians India 2000; 48 (4): $452-$ 3.
30.- Ahn MI, Park SH, Park YH: Poland's syndrome with lung cancer. A case report. Acta Radiol 2000; 41 (5): 432-4.

31.- Ravitch MM: Poland's syndrome. In: Wada J, Yokoyama M,eds. Chest wall deformities and their operative treatment. Tokyo: AD Printing 1990; 20919.

32.- Fukushima T, Otake T, Yashima R, et al: Breast Cancer in Two Patients with Poland's Syndrome. Breast Cancer 1999; 6 (2): 127-30.

33.- Toprak D: Asymmetry of the thorax and hypoplastic hand. Am Fam Physician 2006; 74 (9): 1585-6.

34.- Stevens DB, Fink BA, Prevel C: Poland's syndrome in one identical twin. J Pediatr Orthop 2000; 20 (3): 392-5.

35.- Sujansky E, Riccardi VM, Matthew AL: The familial occurrence of Poland syndrome. Birth Defects Orig Artic Ser 1977; 13 (3A): 117-21.

36.- Soltan HC, Holmes LB: Familial occurrence of malformations possibly attributable to vascular abnormalities. J Pediatr 1986; 108 (1): 112-4.

37.- David TJ: Familial Poland anomaly. J Med Genet 1982; 19 (4): 293-6.

38.- Czeizel A, Vitéz M, Lenz W: Birth prevalence of Poland sequence and proportion of its familial cases. Am J Med Genet 1990; 36 (4): 524.

39.- Samuels TH, Haider MA, Kirkbride P: Poland's syndrome: a mammographic presentation. AJR Am J Roentgenol 1996; 166 (2): 347-8.

40.- Vélez A, Moreno J: Poland's syndrome and recessive $\mathrm{X}$-linked ichthyosis in two brothers. Clin Exp Dermatol 2000; 25 (4): 308-11.

41.- Gorlin RJ: Risk of recurrence in usually nongenetic malformation syndromes. Birth Defects Orig Artic Ser 1979; 15 (5C): 181-8.

42.- Merlob P, Schonfeld A, Ovadia Y, Reisner SH: Realtime echo-Doppler Duplex Scanner in the evaluation of patients with Poland sequence. Eur J Obstet Gynecol Reprod Biol 1989; 32 (2): 103-8.

43.- Bouvet JP, Leveque D, Bernetieres F, Gros JJ: Vascular origin of Poland syndrome? A comparative rheographic study of the vascularisation of the arms in eight patients. Eur J Pediatr 1978; 128 (1): 17-26.

44.- Bamforth JS, Fabian C, Machin G, Honore L: Poland anomaly with a limb body wall disruption defect: case report and review. Am J Med Genet 1992 Jul 15; 43 (5): 780-4.

45.- Puvabanditsin S, Garrow E, Augustin G, Titapiwatanakul R, Kuniyoshi KM: Poland-Möbius 
JIMÉNEZ E. y cols.

syndrome and cocaine abuse: a relook at vascular etiology. Pediatr Neurol 2005; 32 (4): 285-7.

46.- David TJ: Nature and etiology of the Poland anomaly. N Engl J Med 1972; 287 (10): 487-9.
47.- Martínez-Frías ML, Czeizel AE, Rodríguez-Pinilla E, Bermejo E: Smoking during pregnancy and Poland sequence: results of a population-based registry and a case-control registry. Teratology 1999; 59 (1): 35-8. 